\title{
n-FOLD JORDAN PRODUCT COMMUTING MAPS WITH A $\lambda$-ALUTHGE TRANSFORM
}

\author{
YounJIN KIM AND EUNGIL Ko
}

Abstract. Let $\mathscr{B}(H)$ be the set of all bounded linear operators from $H$ to $H$, where $H$ is a complex Hilbert space. In this paper, we study the properties of $T$ when the $\lambda$-Aluthge transform of $T^{n}$ is $T$. Also we prove that the bijective map $\Phi: \mathscr{B}(H) \rightarrow \mathscr{B}(K)$ commutes with a $\lambda$-Aluthge transform under the $n$-fold jordan product if and only if there exists an unitary operator $U: H \rightarrow K$ such that $\Phi(T)=U T U^{*}$ for every $T$ in $\mathscr{B}(H)$.

Mathematics subject classification (2010): 47A05, 47B49, 46L40.

Keywords and phrases: Quasi-normal operators, polar decomposition, $\lambda$-Aluthge transform.

\section{REFERENCES}

[1] A. Aluthge, On $p$-hyponormal operators for $0<p<1$, Integral Equations Operator Theory 13 (1990) 307-315.

[2] F. Botelho, L. Molnar, And G. Nagy, Linear bijections on von Neumann factors commuting with $\lambda$-Aluthge transform, Bull. London Math. Soc. 48 (2016) 74-84.

[3] F. ChaввавI, Product commuting maps with the $\lambda$-Aluthge transform, J. Math. Anal. Appl. 449 (2017) 589-600.

[4] F. CHABBABI AND M. MBEкHTA, Jordan-product commuting nonlinear maps with $\lambda$-Aluthge transform, J. Math. Anal. Appl. 450 (2017) 293-313.

[5] I. JunG, E. KO AND C. PEARCY, Aluthge transform of operators, Integral Equations Operator Theory 37 (2000) 437-448.

[6] K. OKUBO, On weakly unitarily invariant norm and the Aluthge transformation, Linear Algebra Appl. 371 (2003) 369-375. 